\title{
Hacia una nueva conceptualización de la Bioética y aplicación de sus principios en el ordenamiento jurídico
}

Towards a new conceptualization of Bioethics and application of its principles in the legal system

Autor: Brayan Alexander Díaz Piragauta

Para citar este artículo:

Diaz Piragauta, B. A. (2020). Nueva conceptualización de la bioética y aplicación de sus principios en el ordenamiento jurídico. Revista Derecho y Realidad, 18, (36), 239-251. 


\section{HACIA UNA NUEVA CONCEPTUALIZACIÓN DE LA BIOÉTICA Y APLICACIÓN DE SUS PRINCIPIOS EN EL ORDENAMIENTO JURÍDICO*}

Towards a new conceptualization of Bioethics and application of its principles in the legal system

Rumo a uma nova conceituação da bioética e aplicação de seus princípios no sistema jurídico

\section{Brayan Alexander Díaz Piragauta ${ }^{a}$}

resurgence_@outlook.com

Recepción: 3 de agosto 2020

Aceptación: 3 de septiembre 2020

\section{RESUMEN}

El presente artículo pretende evidenciar la forma en la que la bioética ha evolucionado gracias a su aplicación al campo del derecho. Asimismo, conocer como la aplicación de principios bioéticos al ordenamiento jurídico supera la imposición de códigos morales únicos, los cuales influyen en la toma de decisiones para regular, desde el ámbito jurídico, diversos temas de interés. Algunos de ellos son el aborto y la eutanasia, los cuales, para su desarrollo, requieren una ponderación bioética de derechos. Sobre todo, en contextos donde la necesidad de desarrollar una aplicación bioética en las sociedades democráticas del siglo XXI surge por los ordenamientos jurídicos de países pioneros, basados en la participación de ciudadanos autónomos, mediante procedimientos de deliberación en todos los niveles institucionales y sociales. Bajo la metodología de análisis y síntesis doctrinal, jurisprudencial y constitucional, se evidencia como la aplicación de los principios bioéticos a temas puntuales del derecho, tiene como resultado una significativa responsabilidad social que da importancia a los casos y las situaciones jurídicas complejas. Las cuales se dirimen mediante la ponderación de derechos, en coherencia con las evidencias del progreso científico. Teniendo como principal resultado, una síntesis de la relación entre derecho y bioética, llevando a la importancia de una elaboración participativa y abierta de las normas e igualmente la implementación de respuestas jurídicas relativas a cada caso en particular.

\section{PALABRAS CLAVES}

Bioética; Aborto; Eutanasia; Ponderación de derechos.

* Artículo de Investigación

a. Candidato a especialista en Derecho Administrativo de la Universidad Nacional. Abogado - Facultad de Derecho y Ciencias Sociales U.P.T.C. Miembro del Grupo de investigación en Justicia Social "Primo Levi”. Dirección: Calle 19 b. No. 26-47 Duitama - Boyacá 


\section{ABSTRACT}

This article aims to show how bioethics has evolved through its application to the field of law. Likewise, to know how the application of bioethical principles to the legal system overcomes the imposition of unique moral codes, which influence decision making to regulate, from the legal field, diverse topics of interest. Some of them are abortion and euthanasia, which, for their development, require a bioethical weighting of rights. Especially in contexts where the need to develop a bioethical application in the democratic societies of the 21st century arises from the legal systems of pioneering countries, based on the participation of autonomous citizens, through deliberative procedures at all institutional and social levels. Under the methodology of analysis and doctrinal, jurisprudential and constitutional synthesis, it is evident how the application of bioethical principles to specific issues of law, results in a significant social responsibility that gives importance to cases and complex legal situations. These are resolved through the weighting of rights, in coherence with the evidence of scientific progress. Having as main result, a synthesis of the relationship between law and bioethics, leading to the importance of a participative and open elaboration of the norms and the implementation of legal answers related to each case.

\section{KEYWORDS}

Bioethics; Abortion; Euthanasia; Weighting of rights..

\section{RESUMO}

O objetivo deste artigo é mostrar a evolução da bioética a partir de sua aplicação ao campo do direito. Da mesma forma, saber como a aplicação dos princípios bioéticos ao ordenamento jurídico supera a imposição de códigos morais singulares, que influenciam a tomada de decisões para regular, desde o campo jurídico, diversos temas de interesse. Algumas delas são o aborto e a eutanásia, que, para seu desenvolvimento, requerem uma pesagem bioética de direitos. Sobretudo em contextos em que a necessidade de desenvolver uma aplicação bioética nas sociedades democráticas do século XXI decorre dos ordenamentos jurídicos dos países pioneiros, baseados na participação de cidadãos autónomos, através de procedimentos deliberativos a todos os níveis institucionais e sociais. No âmbito da metodologia de análise e síntese doutrinal, jurisprudencial e constitucional, fica evidente como a aplicação dos princípios bioéticos a questões específicas do direito, resulta numa significativa responsabilidade social que dá importância aos casos e às situações jurídicas complexas. Que são resolvidos por pesagem de direitos, em coerência com as evidências do progresso científico. 0 resultado principal é uma síntese da relação entre direito e bioética, levando à importância de um desenvolvimento participativo e aberto das regras e também da implementação de respostas jurídicas relacionadas a cada caso particular.

\section{PALAVRAS-CHAVE:}

Bioética; aborto; eutanásia; ponderação de direitos.

\section{INTRODUCCIÓN}

Bajo la premisa de que el derecho no se refiere únicamente a la función de establecer un marco en donde los individuos interactúen entre sí, reducción ligeramente individualista, sino que también garantizan la función de propender activamente, por la complacencia de los intereses sociales, la consagración de los valores socialmente legitimados. Dichos valores llegan a convertirse en bases de las legislaciones en las sociedades actuales. Tal aspecto genera que en algunos países se desconozca la concepción de derechos exigibles, entendidos desde una visión concreta, específica y garantista. Con lo cual, lograrían diferenciarlos de los intereses populistas netamente basados en dogmas absolutos, despóticos y marginales, aún presentes en las sociedades del siglo XXI.

Por lo tanto, la relevancia de la bioética aplicada al campo del derecho radica en la forma en la cual se considera la perspectiva de varias disciplinas, presentando un análisis socio-jurídico de la forma en que 
se estipulan los principios y valores en las sociedades democráticas, fundamentados en el desarrollo científico, la protección de la persona y sus derechos fundamentales. La importancia de la presente investigación radica en entender la evolución de la bioética para propender por formas de vida más respetuosas con las realidades jurídicas del ser humano. De igual forma, la aplicación de sus principios es útil al valerse de la ciencia y lograr que en el ordenamiento jurídico sólo se regulen aquellas conductas estrictamente necesarias para la vida social, reglada por las instituciones sociales, quienes directamente les asiste una función integradora incluyente y participativa. En tal orden de ideas, la posibilidad de establecer un ordenamiento jurídico con unas bases bioéticas que armonicen con el actuar de los individuos y las características particulares de cada situación.

De acuerdo con lo anterior, en la presente investigación se propone responder ¿cómo es el desarrollo en la aplicación de los principios bioéticos al ámbito jurídico, en temas como el aborto y la eutanasia, de las sociedades democráticas del siglo XXI?

Para dar respuesta al interrogante, resulta necesario abordar el siguiente orden temático: inicialmente se analizará la evolución del concepto de bioética y su evolución en el campo de las ciencias sociales, especialmente su aplicación al derecho. Luego, se evidenciará una extensión de la aplicación de los principios bioéticos a temas relevantes en el ámbito jurídico como lo son el aborto, la eutanasia. Por consiguiente, se sintetiza el impacto de la investigación sobre bioética jurídica. Para finalmente terminar con las conclusiones y los aportes al debate académico.

\section{HACIA UNA NUEVA CONCEPTUALIZACIÓN DE BIOÉTICA APLICADA AL DERECHO}

El término bioética surge como propuesta de una nueva disciplina para relacionar el conocimiento científico, referente a la vida en todas sus expresiones, y la ciencia humanista enfocada desde la perspectiva de la ética.
Por lo tanto, no existe una sola manera de definir la bioética. Sin embargo, es válido argumentar que la bioética se entiende como un campo interdisciplinario que comprende aspectos filosóficos, científicos, sociales, antropológicos, psicológicos, técnicos, legales, etc. Asimismo, fomenta una nueva tendencia social y cultural para redefinir los conceptos que existen respecto a la naturaleza del ser humano, como nacer, reproducirnos, cuidarnos o morir. Si bien tales aspectos tienen una connotación jurídica, de la cual el derecho se instrumentaliza para reglamentar, igualmente implican que la bioética sea una disciplina que propenda por la búsqueda de acuerdos racionales entre personas de diversos colectivos de la sociedad. "Procura armonizar el uso de las ciencias biomédicas y sus tecnologías con los derechos humanos, en relación con los valores y principios éticos universalmente proclamados." (Tintan, 2004, p.10). Aproximadamente, la bioética se puede definir como el análisis sistemático entre las nociones de la moral y la noción de ciencia enfocado hacia la vida con dignidad. La bioética aplicada al derecho sirve como instrumento de materialización de derechos, para circunstancias concretas, a la que se le asigna una finalidad práctica a través de diferentes formas de institucionalización.

Respecto al origen del término, es posible identificar una discrepancia entre dos posturas históricas. Por un lado, hay quienes consideran que el primero en introducir el término fue el teólogo y filósofo alemán Fritz Jahr, quien, en 1927, utilizó el término "Bio-Ethik" en un artículo relacionado con la ética para con las plantas y animales. Por otro lado, se le atribuye al bioquímico y oncólogo Van Rensselaer Potter, quien en el año 1970 usó el término "bio-ethics" dentro de un artículo, para, un año después, publicar un texto titulado "Bioethics: bridge to the future". Entre ambos, Potter presenta mayor popularidad, hay quienes dividen la historia de la Bioética en un antes y después de Potter. La etapa antes de Potter hace referencia al contenido ético en el juramento Hipocrático y los principios de la deontología médica. Después de Potter se tiene como referencia de inicio el final de la segunda 
guerra mundial, hasta 1967, cuando se realizó el primer trasplante de corazón por el médico Sudafricano Christian Barnard.

En el presente punto del debate, es necesario hacer referencia a cómo la noción de bioética se relaciona con varias áreas del conocimiento. En tal combinación surge una corriente que apoya el concepto de la bioética aplicada al derecho que tiene, como base, una noción exclusivamente preventiva, Maldonado (2007) afirma. "Una preocupación, particularmente de algunos sectores interesados en ponerle límites a las investigaciones científicas sobre seres y sistemas vivos, a partir de la normatividad jurídica" (p.43). Es decir, un ordenamiento jurídico que regule la aplicación de nuevas tecnologías con relación a la vida. Centrándose en el estudio de los peligros y amenazas que la biomedicina, en particular las nuevas tecnologías aplicadas a los seres $\mathrm{y}$ sistemas vivos y las consecuencias que pueden ocasionar, es necesario apoyarse concomitantemente en la investigación científica y el aporte de las ciencias humanistas como el derecho. Principios jurídicos como la dignidad, la justicia, la libertad o autonomía de la voluntad, la igualdad, el respeto, entre otros, se identifican plenamente con los principios de la bioética, ofreciendo un factor común ineludible para su estudio. La ponderación de derechos, por ende, los principios de autonomía, no maleficencia, beneficencia y justicia, materializan la lucha por los derechos, por la distribución justa de los recursos y el control de las tecnologías que confieren el dominio sobre la vida. Perfilan su contexto definiendo los retos de la bioética. "Deliberando no sobre lo materialmente bueno sino sobre lo intersubjetivamente justo. Rechazando el absolutismo, el dogmatismo, el fundamentalismo, el relativismo y el subjetivismo, no se compromete con ninguna moral, religión o política concretas y se presenta como una ética aplicada a las interpelaciones de nuestro mundo" (Camps, 2013, p. 392 y ss).

Es necesario establecer la relación entre bioética y derecho para entender la aplicación de los principios bioéticos al ordenamiento jurídico. Con relación a ello, Rivera (2011) afirma:

Son tres los puntos de conexión entre ambos: la conexión práctica: se da porque el derecho opera como un límite para la bioética, los médicos, miembros de los comités de bioética, investigadores, etc. deben ajustar sus recomendaciones y decisiones a lo que dice el ordenamiento jurídico del lugar donde ejercen sus funciones. Existe una conexión legislativa, en tanto que la bioética brinda razones y fundamentos a los legisladores a la hora de crear o modificar normas de esa materia. Por último, la conexión interpretativa, se da ante la necesidad de solucionar los inconvenientes propios del derecho (textura abierta, colisión de principios y derechos, etc.) mediante la actividad interpretativa. Para ello, los juristas $\mathrm{u}$ operadores del derecho recurren a estándares extrajurídicos, como son los de la ética en general y la bioética en particular para encontrar la respuesta correcta a la cuestión controvertida (p.75).

El nuevo concepto de bioética, aplicado al derecho, cumple una función orientadora y práctica que promueve la deliberación y sustenta una razón dúctil, que conjuga la generalidad y abstracción de los principios con la singularidad del caso. Una razón práctica y compasiva que procede con precaución, otorgando relevancia a los derechos y ponderando los principios según cada caso en particular, frente al pragmatismo y el relativismo moral, sostiene la objetividad de los valores y dialoga para sentar las bases éticas políticas y jurídicas de las sociedades bien fundamentadas, que poseen una concepción compartida de la justicia. Asimismo, deja al sujeto decidir y actuar, confiere la facultad de adoptar principios válidos para intervenir en las decisiones del caso concreto, materializando la conexión interpretativa entre bioética y derecho, generando directamente los fundamentos para la conexión legislativa, la cual generalmente es guiada por la moral 
mayoritaria de cada sociedad democrática del siglo XXI.

Las bases de dicha relación se reconocen en los derechos humanos, que han surgido de la conciencia social de la humanidad. La declaración universal de bioética y derechos humanos de 2005, que se ocupa de la ética, la medicina, las ciencias de la vida y las tecnologías aplicadas a los seres humanos, da un marco universal de principios de la bioética como guía para los estados. De igual forma, establece expresamente que, en cualquier tipo de prácticas y decisiones vinculadas con las ciencias de la vida y con la biotecnología, los derechos humanos hacen parte del estado social de derecho que se propone garantizar estándares mínimos de salario, salud, habitación y educación para todos. Siguiendo así, la idea de derechos y no de simple voluntad del estado, con fundamento en la seguridad jurídica de la existencia de nuevos derechos fundamentales $y$ efectivos mecanismos de protección, democracia participativa y control político.

\section{APLICACIÓN DE PRINCIPIOS BIOÉTICOS A CASOS JURÍDICOS RELEVANTES}

Desde diferentes perspectivas, un amplio consenso sustenta el sentido del "derecho como una práctica social que incorpora la pretensión de corrección" (Atienza, 2013, p. 29). La corrección, como finalidad de la bioética en el derecho, no consiste en la aplicación mecánica de principios o de normas morales programadas previamente. Por el contrario, en la aplicación de los campos argumentativos de acción, es relevante el proceso deliberativo, como un espectro de diálogo multidisciplinario y pluralista, con garantías mínimas para identificar los aspectos relevantes por considerar. De tal modo, la acción práctica en determinadas circunstancias responde a los valores en cuestión de cada individuo, "surgiendo desde el respeto radical a la dignidad de la persona" (Ballesteros, 2007, p. 110). Dicho objetivo no se consigue con la simple aplicación de principios generales ampliamente aceptados, sino que es necesario contemplar los principios morales de cada sociedad democrática como el producto condensado de experiencias y de circunstancias que han ido cambiando, a lo largo de generaciones, plasmadas en el ámbito jurídico.

Los principios morales son referentes de instituciones, grupos y sociedades enteras, interfiriendo en procesos esenciales de socialización y avance científico. Allí surge la importancia de la bioética, puesto que se desarrolla bajo la garantía del laicismo constitucional como una manera de evitar que la moral sea definida por dogmas religiosos. "Para considerar los principios morales en el ordenamiento jurídico, se exige construir reglas y garantizar los derechos y considerarlos no ya como principios programáticos, sino como reglas de pleno derecho que informan el ordenamiento, contienen los criterios de reconocimiento y aplicación que vinculan a los operadores jurídicos" (Pérez Luño, 1993, p. 455). Los principios de la bioética, aplicados al derecho, son guías de acción para tomar decisiones frente a situaciones que generan una contraposición de derechos, tanto individuales como colectivos, relacionadas directamente con el desarrollo de la vida, "se comprometen con los derechos humanos" (Pérez Luño, 2007, pp. 231-235), en todas sus manifestaciones y planos: la naturaleza, las personas y los ámbitos social, político, jurídico y económico. Claro está, analizando esos problemas, interpretándolos y planteando si bien no respuestas absolutas, por lo menos procedimientos que aporten soluciones para garantizar mínimos de imparcialidad y pensamiento crítico de cada situación.

Tom Beauchamp y James Childress, en 1979, plantearon cuatro grandes principios bioéticos: autonomía, beneficencia, no maleficencia y justicia. Para desarrollar el objetivo de la presente investigación es necesario extender la conceptualización, aplicación y evolución de los principios directamente al derecho sustancial, propio de las sociedades democráticas del siglo XXI. Para resolver los conflictos que aparecen al momento de tomar decisiones en el ámbito 
jurídico, surge la bioética de orientación basada en conseguir un método sistemático de reflexión que permita elegir una solución correcta ante un dilema bioético.

Sus principios no son absolutos y rígidos sino dúctiles y equitativos. Desde la autonomía y el pluralismo, conjuga la convicción de los principios y la responsabilidad de las consecuencias que en una sociedad plural, democrática y abierta configuran lo justo que no siempre coincide con lo bueno (Feito, 2009, pp. 27-99).

Para detallar tal situación, se hace necesario ampliar la aplicación de los principios de la bioética en temas de relevancia social de los cuales el derecho debe regular. Extendiendo el significado de cada concepto en una aplicación práctica del derecho, puntualmente en el tratamiento que las actuales sociedades han desarrollado respecto a temas con trascendencia social, que implican necesariamente la ponderación de derechos fundamentales.

\section{PRINCIPIO DE AUTONOMÍA Y PRINCIPIO DE BENEFICENCIA}

El principio de autonomía refleja la capacidad de la persona de tomar decisiones por sí misma, sin influencia externa, por su autodeterminación y su privacidad en la toma de decisiones. Tal principio, desde el punto de vista bioético, se materializa con el consentimiento informado del paciente, como su derecho y deber del profesional que lo atiende. En tal orden de ideas, la voluntad del paciente debe ser respetada como la más importante garantía a cumplir. "el principio de autonomía aparece como un reclamo al respeto por las conductas autorreferentes, lo que implica la no intervención estatal en el plan de vida que cada persona, cada ciudadano elige; y reconociendo como único límite no dañar a terceros"(Mainetti,2008, p.68).

La beneficencia es entendida como el deber, del profesional de salud, de actuar en pro del beneficio al paciente y sus familiares. Así, se legitima el actuar en la medida que se propenda por el bienestar del paciente, en ocasiones el beneficio del paciente puede ser diferente a su voluntad, el cual se debe ponderar en busca del consenso por una solución efectiva. En casos de urgencia y sin posibilidad de intervención de los familiares, el médico está obligado a actuar defendiendo los mejores intereses del paciente, como él los entiende, a partir del conocimiento que la medicina le provee ${ }^{1}$. Una extensión de dicho concepto, que inicialmente tiene aplicación en la rama de la medicina, al desarrollo en el ordenamiento jurídico, puntualmente el derecho de autodeterminación en el aborto.

Las posturas respecto al aborto inducido se han caracterizado de forma general por quienes por una parte otorgan al cigoto, en todas sus etapas, una protección absoluta basados en el derecho a la vida, y por quienes confieren a la mujer una protección absoluta de su autodeterminación para decidir sobre su cuerpo, de estas posturas se genera un debate que ha trascendido por varias décadas. Los opositores del aborto defienden el derecho a la vida del feto. Pero ese "derecho del feto a la vida es relativo y no absoluto en la medida en que es la madre la que lo otorga, y solamente puede ser abolido por los derechos de la madre." (Ferrater, 1983 pág. 42). Los principios morales sirven como marcos de referencia para la aplicación de determinados tratamientos en el ámbito jurídico. Entre ellos, la penalización del aborto, no obstante, frecuentemente entran en conflicto entre sí y cada uno de ellos puede ser ponderado de diversas formas según el contexto en que se desarrollen.

Desde el punto de vista jurídico, argumentar que existen derechos cuya protección es absoluta es una falacia, puesto que todos los derechos tienen sus límites ante la colisión de derechos que deben ser ponderados para no vulnerar derechos propios o de terceros. Porque, así como no hay derechos absolutos sino resistentes (Prieto, 1990, p. 83), tampoco hay principios absolutos. En un sector muy conservador de la sociedad se cree erróneamente que el

1. Viada González CE, Ballagas Flores C, Blanco López Y. Ética en la investigación con poblaciones especiales. Rev Cubana Invest Biomed. 2006;20(2):140-9. 
derecho a la vida es el más importante y se encuentra por encima de los demás derechos fundamentales del individuo. Para refutar tal tesis es necesario apelar a la teoría de que todos los derechos fundamentales por naturaleza no tienen jerarquía, son indivisibles, interdependientes y se complementan mutuamente ${ }^{2}$. La bioética establece mediante el principio de autonomía y de beneficencia un marco referencial aplicable para interpretar el aborto inducido como una conducta aceptable tanto para la mujer, el cigoto en formación, y la sociedad como garante de derechos.

Científicamente, conocemos que el embarazo es un proceso con diversas fases del desarrollo embrionario. En la primera fase, que va hasta las 12 primeras semanas de gestación, el óvulo fecundado, denominado cigoto, no llega necesariamente a convertirse en un feto que potencialmente nazca vivo, esto es porque pueden convertirse en tejidos amorfos, tumores o fetos anencefálicos. Apenas a los 14 días empieza a insinuarse la columna vertebral, lo cual indica que el feto no tiene un sistema nervioso que le permita "sentir dolor o estar consciente” (Singer, 2003 pág. 108). Por ende, desde el punto de vista científico, el ser humano puede catalogarse como tal cuando tiene la autonomía fisiológica necesaria para experimentar funcionalidad y conciencia de los estímulos externos. Dicha condición se logra a partir del último trimestre del embarazo. Tampoco hay una razón clara para que una entidad, la cual apenas potencialmente puede ser persona, deba tener tanto reconocimiento como una persona cabal: "el feto no es formalmente una persona, aun cuando se acepte que posee una naturaleza humana virtual" (Vázquez, 2009 pág. 54). Esta controversia no puede ser resuelta por el derecho privilegiando una determinada tesis moral, "la que considera al feto una persona, imponiéndose a todos y por tanto obligando también a las mujeres que no la compartan a sufrir dramáticas consecuencias." (Ferrajoli, 2006, p.37).

2. M. Piekarewicz Sigal, Bioética, aborto y políticas públicas en América Latina, Barcelona, 2015.
En este orden de ideas, si bien la mujer es reconocida como sujeto titular de derechos, el cigoto, reconocido como un bien jurídicamente tutelado que goza de la protección propia otorgada al genoma humano, en tanto patrimonio genético goza de intangibilidad e integridad, salvo por motivos terapéuticos: la penalización del aborto en todas las semanas de gestación surge como una medida desproporcionada que desconoce los fundamentos científicos. La utilidad de la ciencia al servicio del derecho y el principio de autonomía y beneficencia propios de la bioética permite que la mujer goce de la potestad para decidir sobre la continuidad del embarazo, dentro de los términos que la ciencia permite catalogar como cigoto al óvulo fecundado. Así, la protección es variable de acuerdo con el tiempo de fecundación.

La penalización de la interrupción voluntaria del embarazo es contraria, fundamentalmente, a las recomendaciones de organismos internacionales sobre la materia. Entidades autorizadas de las Naciones Unidas han llegado a la conclusión de que las mujeres tienen el derecho de decidir en los asuntos relacionados con la interrupción voluntaria del embarazo, inscribiéndose como un derecho reproductivo, al ponderar el resto de los derechos humanos que corren riesgo cuando el acceso de las mujeres a los servicios de aborto legal y seguro está restringido. Siguiendo a Cohen (1999), "forzar a una mujer a soportar un embarazo no deseado es imponer por la fuerza una identidad: la identidad de ser mujer embarazada y de madre. Innegablemente, es la integridad corporal de las mujeres, en el sentido físico, tanto como el emocional, lo que se pone en juego a través de la penalización del aborto. Pero también se hace peligrar su integridad personal" (p.226).

En síntesis, la Bioética, mediante el principio de autonomía y de beneficencia, permite materializar su utilidad para transformar el ordenamiento jurídico y la forma en que las sociedades democráticas del siglo XXI implementan la aplicación de derechos fundamentales a casos como 
la interrupción voluntaria del embarazo, la cual debe estar libre de influencias políticas, religiosas y económicas: "De ahí la separación entre el Derecho y la moral no para desmoralizar el Derecho, sino para deslegalizar la moral e impedir que el Estado interfiera en asuntos que sólo y exclusivamente solo competen al sujeto" (Laporta, 1993, pp. 59 y 107). El acto de reconocer en la capacidad de autodeterminación del ser humano un principio que cumple con mayores garantías en la dimensión moral de la sociedad como la base para respetar la voluntad de la mujer es algo peligroso, cuando científicamente se prueba que dicha voluntad no vulnera derechos ajenos, no solo en materia médica, sino en general en todas las disciplinas que convergen y se relacionan con el aborto inducido.

\section{PRINCIPIO DE NO MALEFICENCIA Y PRINCIPIO DE JUSTICIA EN LA EUTANASIA}

El principio de no maleficencia, desde el punto bioético, se fundamenta en no cometer acciones que lesionen al paciente, perjudicándolo innecesariamente $0 \quad$ sin justificación, en consecuencia, con los fines perseguidos en función de su bienestar. Es por esto por lo que el profesional se debe comprometer a tener una formación fundamentada en conocimientos sólidos y científicos. Basados en la anterior premisa, es necesario reformular para el caso puntual de la eutanasia, ¿qué se entiende como el bienestar del paciente? Este principio consagra una coacción negativa de no menoscabar el bienestar de un tercero. De esta manera, automáticamente, este concepto adquiere un sentido en el cual la prioridad no es evitar la muerte, sino evitar que su bienestar como ser humano se vea menoscabado directa o indirectamente por el actuar del personal de salud: "Es responsabilidad de los médicos entender sus anhelos, creencias o preocupaciones, y explicar con claridad la situación única que están viviendo, las opciones de manejo y tratamiento, así como hacerles conocer las consecuencias posibles del camino que elijan (Becca, 2005 p.133). Es necesario enfatizar en cómo las sociedades materialmente inclusivas y pluralistas han tenido que desarrollar procedimientos eficaces para hacer frente a los conflictos de interpretaciones sobre el alcance, la pertinencia y la prioridad de la vida con dignidad, igualmente con una forma racional de entender la muerte.

El principio de justicia en la eutanasia se materializa en el deber de actuar del profesional de la salud en el campo médico. Esto es garantizar el acceso a todos los servicios médicos aprobados por el método científico. Igualmente, este principio es extensivo a los sujetos en el mundo del derecho, porque la aplicación para la eutanasia, las decisiones deben ir más allá de los convencionalismos religiosos que vetan de sus concepciones este tipo de prácticas, a causa de que se tiene que las decisiones en materia de políticas públicas para entender una vida con calidad son responsables por vía del acuerdo intersubjetivo: "una persona de cualquier sociedad ha sido tratada justamente si es tratada de acuerdo con lo que es justo, debido o merecido". (Zalvi, 2001, p.55). Asimismo, el deber de cuidado se ve materializado en la obligación de las instituciones que prestan el servicio de estar capacitadas, no solamente en términos de la práctica médica, sino también en términos administrativos de evaluación, diagnóstico y mejoramiento de los procesos para mantenerlos en constante renovación y vigencia.

En 2009, el tribunal supremo de Canadá declaró la inconstitucionalidad de los artículos. 14, 21, 22, 222 y 241 de la Carta Política, y anuló la prohibición del suicidio médicamente asistido. Esta sentencia se considera referente y liberal porque reafirma la dignidad y la autonomía en oposición al paternalismo, y "distingue entre la ética pública y la ética privada y reafirma desde el paradigma autonomista" (Gracia, 2004, pp. 397). Esta es una sentencia relevante porque frente a los objetivos y directrices políticas se toma en serio los derechos, es principialista e impulsada por la integridad porque atiende a los principios que contienen exigencias de justicia y "se imponen sobre las reglas y dan 
la respuesta correcta”. (Pérez Luño, 2009, p. 466). Los principios morales no pueden vulnerar derechos. Es viable reconocer que existe un consenso respecto a la vida como un presupuesto necesario para ejercer los demás derechos, pero se reitera la necesidad de entender la vida como un derecho que no es absoluto, cada individuo puede decidir si continúa o no viviendo cuando las circunstancias que rodean su experiencia no la hacen deseable ni digna de ser vivida.

La eutanasia como concepto holístico debe tener en cuenta el proceso que el paciente ha tenido durante el avance de su enfermedad y su autodeterminación como base para respetar su voluntad, la cual debe tener un límite en los comités científicos cuyos parámetros se basan en el soporte científico. Lo anterior le permite a la bioética servir de base para tomar decisiones que respeten el ya conocido consentimiento informado, como máxima expresión para garantizar el verdadero bienestar del paciente. El principio de no maleficencia y de justicia adquieren una connotación crítica porque reflexionan y cuestionan un problema real, cotidiano y concreto, como lo es enfrentar la muerte. No se puede ofrecer un conjunto de respuestas simplistas, de dogmas rígidamente preestablecidos o fórmulas hechas que indiquen lo que está bien y lo que está mal con la eutanasia. Aunque la muerte es dolorosa cada caso es particular, el bienestar del ser humano se entiende como un concepto dinámico y subjetivo para cada caso. Es por esto por lo que la muerte no puede ser catalogada objetivamente como negativa, el criterio objetivo de reconocer la muerte es inevitable en determinados casos y por lo tanto se debe contar con sistemas jurídicos que regulen el derecho a morir con dignidad.

El derecho a elegir es lo que debe primar ante las situaciones extremas donde se vulneran el bienestar de un paciente. Desde la perspectiva de un enfermo terminal la eutanasia debe ser vista como la conclusión lógica de un proceso de raciocinio donde, basados en un diagnóstico médico, se compara los pros y los contras de vivir con la irreversibilidad o la imposibilidad de recuperación. Los sistemas médicos y jurídicos deben enfocarse a proporcionar al paciente la mejor calidad de muerte posiblemente realizable, en armonía con el derecho de los familiares de estar preparados y conocer la motivación de cada situación, para de esta manera evitar prolongaciones innecesarias que limiten la efectividad de otros derechos diferentes al derecho a la vida.

Desde un punto de vista pluralista, la búsqueda y el respeto por el bienestar del individuo que tiene una condición médica irreversible le confiere una capacidad de asumir, a él o sus familiares, en forma responsable y autónoma, las decisiones sobre la materialización de la dignidad en sus vidas: "Es así como entre la bioética y lo jurídico se produce una fructífera relación por la mediación de la filosofía de los derechos humanos, a partir básicamente del reconocimiento expreso de la dignidad humana como principio fundante común a estas disciplinas" (Hofft, 1997, p.7).

El Estado, a través del derecho, no puede forzar a los ciudadanos a continuar viviendo cuando por las circunstancias extremas en que se encuentran no lo estiman compatible con su propia dignidad. Por el contrario, al Estado le asiste el deber de proteger la vida en consonancia con el respeto a la dignidad humana y al libre desarrollo de la personalidad, no como una respuesta absoluta, sino como una conclusión particular de los principios bioéticos aplicados al ordenamiento jurídico.

\section{IMPLICACIONES DE LA INVESTIGACIÓN SOBRE BIOÉTICA APLICADA AL DERECHO.}

La investigación sobre bioética no puede articular reflexiones pluralistas sobre planteamientos exclusivamente deontológicos en el derecho. Lo idóneo es que se consideren los principios y derechos como relativos en el sentido que han de ser tenidos en cuenta siempre y cuando una circunstancias determinada no permita que los principios se desarrollen de forma conjunta sin afectarse mutuamente, 
implementando, con esto, criterios de aplicación orientados a salvaguardar los valores que dan contenido a los principios en colisión, procurando, también, que las acciones a implementar sean coherentes con la importancia relativa de los derechos por proteger en cada caso específico. En consecuencia, siendo los principios materia prima esencial para la argumentación e interpretación del derecho, y para la solución de casos complejos como los que se han mencionado, "al derecho le asiste una conexión metodológica con la bioética, como ponderación de principios contrapuestos y aplicación a casos concretos, tal y como ha sido expuesto por parte de juristas y de filósofos ocupados con el diálogo entre bioética y derecho". (Francesco Viola, 2009, p.38).

Es relevante precisar que es en el proceso de deliberación donde se identifican los criterios para aplicar principios que fundamentan los derechos que colisionan entre sí, con el fin de procurar que la acción finalmente elegida refleje los valores que dotan de contenido a los principios. Es decir, no se trata simplemente de "hacer lo correcto" porque aparentemente se tiene en cuenta una regla o principio ampliamente aceptado, en ocasiones por mayorías, sino de garantizar en lo posible "el resultado sea justo", atendiendo a las circunstancias y a los detalles relevantes de la situación. Estas características sirven como sustento en la argumentación orientada para identificar qué implementación de la acción es más razonable. "La argumentación reconstruye los puentes entre los hechos y los valores, justifica el razonamiento y pronuncia la única respuesta correcta" (Atienza, 2010, p. 76). Quienes otorgan un carácter absoluto a los principios morales y los consideran válidos pese a las circunstancias, generalmente desarrollan estilos de argumentación moral con rigidez deductiva.

El progreso de las sociedades democráticas del siglo XXI se caracteriza porque tiene presente que no es posible limitar el desarrollo científico y jurídico de forma arbitraria, obedeciendo a costumbres netamente dogmáticas. Sin embargo, tampoco se puede aceptar un desarrollo científico que por sí mismo atente con la existencia y estabilidad de la humanidad, motivo por el cual la bioética se está consolidado como un complemento del derecho para regular las nuevas tecnologías y la biomedicina, valiéndose de herramientas interpretativas para ejercicio de derechos en todas sus categorías, generando una fundamentación en el estudio de los riesgos, peligros y amenazas que el avance en estas áreas pueda implicar para el ser humano. Por lo tanto, todo aquello que ponga en riesgo la vida, la libertad, la dignidad y los demás principios característicos de una sociedad contemporánea, democrática y participativa, se vuelve objeto de regulación normativa y análisis jurídico.

En la aplicación de este precepto se genera una consecuencia respecto a los principios que se consideran fundamentales para el desarrollo y estabilidad de la sociedad. Para un sector el valor fundamental es la vida, para otro el valor fundamental es la autodeterminación, $y$, generalmente, la posición que se tiene suele inclinar las percepciones sobre la aplicación de la bioética en las sociedades contemporáneas del siglo XXI. La bioética aplicada al derecho es esencial para la eficaz toma de decisiones y la creación de políticas públicas que respondan a la complejidad de cada situación, Potter (2002) afirma: "la acción tendrá que ser constreñida y guiada por el conocimiento biológico mientras emerja la bioética y sea finalmente convertida en acuerdos voluntarios, o leyes reguladoras en instancias específicas" (p.56)

La investigación sobre bioética aplicada al derecho propende por el diálogo entre todos los interesados, con el fin de llegar a fórmulas cada vez más adecuadas, articulando las perspectivas de todos los implicados, haciendo que sus voces sean oídas, fomentando el respeto y el entendimiento mutuo, actuando como referentes en casos y situaciones confusas, y suministrar métodos de análisis y deliberación sobre problemas concretos, basados en la argumentación y el diálogo interdisciplinar, partiendo de la ciencia como una herramienta que 
permite dimensionar que todos los derechos no pueden catalogarse como absolutos, imperativos e imperiosos de manera abstracta.

\section{CONCLUSIONES}

La nueva conceptualización de bioética aplicada al derecho estipula una ética de la responsabilidad abierta, aproximativa y probabilista, capaz de evaluar los riesgos, los costos y los beneficios, según el método científico aplicado al ordenamiento jurídico, haciendo eco de la necesidad de fijar normas que respeten al ser humano, no solo frente a un poderío tecnológico, sino ante las imposiciones y barreras ideológicas que pueden predominar en los sectores conservadores de las sociedades democráticas del siglo XXI. Igualmente, es inclusiva al promover la diversidad y reconocer que todas las opiniones son válidas, enfocándose en reconocer la importancia de concretar los argumentos que se compartan desde las diferentes posturas, por lo que la pluralidad es entendida como un principio rector en el desarrollo de los debates surgidos. $\mathrm{Al}$ tener mayor número de puntos de vista, se puede garantizar en un mayor grado que la síntesis lograda sea eficiente respecto a los participantes del debate. Desde el punto de vista estatal se legitima la creación de políticas públicas mediante la participación de los directamente interesados, generando directamente una efectividad en su implementación.
A pesar de su reciente existencia en el mundo de la ciencias biológicas y jurídicas, la bioética ha logrado una aceptación considerable en todos los ámbitos de aplicación tanto del derecho como de la medicina. Ha servido para fundamentar desde un punto de vista ético la investigación con seres humanos. Ha contribuido a transformar el modelo paternalista de relación con los pacientes, y ha cambiado significativamente la forma de tomar decisiones médicas y sociales, especialmente a interpretar los principios bioéticos para regular determinadas conductas en el mundo jurídico como el aborto inducido, la eutanasia, desde un fundamento holístico, consensual, laico y argumentativo.

En el campo de la investigación sobre bioética aplicada al derecho se concluye cómo la noción de derechos en colisión se ha extendido hacia una visión más realista de nuestras sociedades, llegando a abarcar una nueva concepción más dinámica de los valores y principios, todo tipo de problemas relacionados con la vida, generando nuevos debates y retos sobre cómo se perciben las interacciones entre los sujetos titulares de derechos y obligaciones. Esto abre paso a un cambio de paradigma de pensamiento más inclusivo, participativo y analítico de la materialización de principios bioéticos aplicados a los casos específicos del aborto inducido y la eutanasia, interpretados $\mathrm{y}$ aplicados mediante el derecho en las sociedades democráticas del siglo XXI.

\section{REFERENCIAS BIBLIOGRÁFICAS}

» Atienza, M. (2012). El sentido del Derecho. Barcelona: Ariel Derecho.

» Atienza, M. (2013). Curso de argumentación jurídica. Madrid: Trotta.

» Ballesteros, J. (2007). Sobre el sentido del Derecho. Madrid: Tecnos.

» Bobbio, N. (1991). El tiempo de los derechos. Trad. de Rafael de Asís Roig. Madrid: Sistema.

» Bouesseau, M (2005). La muerte como frontera de sentido: fundamentos para la elaboración de una ética de la medicina paliativa. Boston: Ars Medica.

» Camps, V. (2013). Breve historia de la Ética. Barcelona: RBA.

» Tinant, E. (2004). Antología para una bioética jurídica. Buenos Aires: La Ley. 
» Cohen, J. (1999). Para pensar de nuevo la privacidad, la autonomía, la identidad y la controversista sobre el aborto. Debate Feminista (19).

» Feito, L. (2009). Ética y enfermería. Madrid: UPC.

» Ferrajoli, L. (2008). "La cuestión del embrión entre el derecho y la moral”, en Democracia y garantismo, trad. P.A. Ibáñez (págs. 153-172). Madrid: Trotta.

» Ferrajoli, L. (2011). Constitucionalismo principialista y constitucionalismo garantista. México: Doxa.

» Gracia, D. (1989). Fundamentos de bioética. Madrid: Eudema.

» García, G. (2002). Comp. Bioética e Investigación Científica. Memorias Primer Congreso Internacional. Universidad Militar Nueva Granada. Bogotá.

» Hofft, P. (1999). Bioética y derechos humanos. Temas y casos, Buenos Aires: KJH

» Maldonado, C. (2007). Bioética, biopolítica y bioeconomía: panorama práctico sobre la bioética. Bogotá: Temis.

» Mainetti, J. A. y M. M. El amparo de la bioética cuando ser madre resulta un drama. Lexis-Nexis $\mathrm{N}^{\circ} 983734$.

» Pérez Luño, A.E. (2007). Trayectorias contemporáneas de la Filosofía y la Teoría del Derecho. Madrid: Tébar.

» Piekarewicz Sigal, M. (2015). Bioética, aborto y políticas públicas en América Latina. Revista de Bioética y Derecho (No. especial), 210 - 219.

» Rivera, E. (2011). Derecho y Bioética, en Problemas de vida o muerte. Madrid: Marcial.

» Singer, P. (2003). Ética práctica. Madrid: Cambridge University Press.

» Tinant, E. (2003). Los derechos humanos a la luz de la bioética, J.A. 2003-III, fascículo $\mathrm{N}^{\circ} 4$, p. 88.

» ZALVI, Mauricio. Justice (Príncipe de). En: HOTTOIS, Gilbert. 2001. 